\title{
Paul CESBRON et Yvonne KNIBIEHLER, La Naissance en Occident, Paris, Albin Michel, 2004, 363 p.
}

\section{Marie-France Morel}

\section{(2) OpenEdition}

\section{Journals}

\section{Édition électronique}

URL : https://journals.openedition.org/clio/1483

DOI : 10.4000/clio.1483

ISSN : 1777-5299

Éditeur

Belin

\section{Édition imprimée}

Date de publication : 1 avril 2005

Pagination : 303-304

ISBN : 2-85816-781-8

ISSN : 1252-7017

\section{Référence électronique}

Marie-France Morel, «Paul CESBRON et Yvonne KNIBIEHLER, La Naissance en Occident, Paris, Albin Michel, 2004, 363 p. », Clio. Histoire, femmes et sociétés [En ligne], 21 | 2005, mis en ligne le 06 juin 2005, consulté le 23 avril 2022. URL : http://journals.openedition.org/clio/1483 ; DOI : https://doi.org/ $10.4000 /$ clio. 1483

Ce document a été généré automatiquement le 23 avril 2022.

Tous droits réservés 


\title{
Paul CESBRON et Yvonne KNIBIEHLER, La Naissance en Occident, Paris, Albin Michel, 2004, $363 \mathrm{p}$.
}

\author{
Marie-France Morel
}

1 Voici un livre à deux voix, où s'entrelacent en un dialogue (rendu sensible par des typographies contrastées) les réflexions d'une grande historienne de la maternité et celles d'un gynécologue obstétricien d'aujourd'hui. Il s'agit de comprendre les profondes transformations de la procréation et de la naissance dans nos sociétés en revenant sur l'histoire longue des croyances et des pratiques autour de la grossesse et de l'accouchement. Même s'il n'est pas question de proposer un retour à l'ancien temps, les deux auteurs sont convaincus qu'une bonne connaissance des symboles et des rites d'autrefois permet de ne pas réduire la naissance d'aujourd'hui à sa seule dimension biologique et médicale. C'est la confrontation des points de vue des deux auteurs qui fait la richesse du livre : si Yvonne Knibiehler a plus à dire sur le temps de l'histoire, Paul Cesbron ne s'interdit pas d'intervenir aussi à propos des pratiques anciennes; et vice-versa, car l'historienne est aussi une actrice engagée dans les évaluations critiques des mutations de notre temps et dans les propositions d'avenir.

2 Le lecteur est donc convié à un parcours historique de l'Antiquité à nos jours. La première partie (Les héritages, le temps du sacré) parcourt à grands traits les sociétés antiques, médiévales ou modernes dans leurs rapports multiples à la fécondité, à la grossesse et à la naissance. La deuxième partie (La médicalisation, le temps de la science), qui part du XIXe siècle, montre comment, à partir du moment où les médecins sont intervenus dans la parturition, on est passé de la naissance à la maison entre femmes (avec ses recours et ses aléas) à l'hyper médicalisation actuelle. Pour le XXe siècle, les précisions techniques données par Paul Cesbron sur les nouvelles techniques, leur histoire et leurs applications sont bien venues, qu'il s'agisse, par exemple, des différentes générations de pilules, des usages de l'échographie et de la péridurale, des 
différentes formes de procréations médicalement assistées ou de diagnostic prénatal. La troisième partie (Perspectives, le temps des questions), qui nous projette dans l'avenir, insiste sur les contradictions entre les exigences des familles d'aujourd'hui qui veulent faire de la naissance une affaire privée et intime et les impératifs à la fois financiers et sécuritaires des politiques de santé publique.

Ce livre est un ouvrage militant qui défend les femmes. Il est parfois un peu irritant quand il en fait d'éternelles victimes : « la vieille culture d'oppression des femmes » (p. 123) ou les «vieux dispositifs patriarcaux d'oppression des femmes" (p. 248) reviennent comme un refrain, comme si les femmes, toujours soumises et exploitées, n'avaient jamais réussi à s'affirmer dans leurs grossesses et accouchements. En fait, sans même insister sur la toute-puissance que leur donne le fait d'être seules à pouvoir enfanter, il est avéré qu'elles ont toujours gardé un pouvoir sur la conduite de leurs couches tant que celles-ci se déroulaient à domicile entre femmes. Même quand elles ont été encouragées par les médecins à aller accoucher à l'hôpital, elles l'ont souvent vécu comme un progrès vers plus d'hygiène et de sécurité (beaucoup y ont tout simplement gagné la vie!) ; n'oublions pas que certaines l'ont aussi apprécié comme un temps de repos et de prise en charge qui pouvait s'apparenter à des vacances. Le livre est plus convaincant quand il fait des propositions qui visent à respecter davantage les femmes: la contraception devrait être mieux individualisée et permettre à chaque femme d'être à l'écoute des rythmes de son corps; la grossesse ne devrait pas être un temps d'intrusion dans l'intimité de la relation entre la mère et son foetus, comme l'est trop souvent l'échographie; lors de l'accouchement, beaucoup de techniques invasives (comme la péridurale, l'épisiotomie, la section précoce du cordon ombilical) ne sont pas aussi nécessaires que les médecins ont réussi à le faire croire aux femmes; beaucoup d'accouchements pourraient se dérouler plus simplement avec un minimum d'assistance médicale (que les sages-femmes, très valorisées dans le livre, sont sans conteste les plus aptes à fournir) et l'accouchement physiologique en maison de naissance ou à domicile devrait être possible pour une grande partie des parturientes. En conclusion du livre, il apparaît que les femmes n'ont pas encore nettement pris conscience que, sous prétexte de sécurité, le pouvoir médical actuel leur a confisqué une partie du vécu de leurs grossesses et de leurs accouchements. Les deux auteurs souhaitent qu'à l'image de celles qui ont su faire pour gagner le droit d'avorter, les femmes se mobilisent pour obtenir de donner la vie comme elles l'entendent. Pour le moment, seules quelques associations peu nombreuses militent en ce sens. C'est certainement là un des nouveaux défis du féminisme contemporain. 